Nouvelles perspectives en sciences sociales

Revue internationale de systémique complexe et d'études relationnelles

\title{
La complexité d'un phénomène linguistique est-elle toujours source de difficulté ? Cas de l'acquisition des relatives en langue première (français et allemand)
}

\section{Claire Martinot}

Volume 9, numéro 1, novembre 2013

Sur le thème : linguistique et complexité

URI : https://id.erudit.org/iderudit/1024040ar

DOI : https://doi.org/10.7202/1024040ar

Aller au sommaire du numéro

\section{Éditeur(s)}

Prise de parole

ISSN

1712-8307 (imprimé)

1918-7475 (numérique)

Découvrir la revue

Citer cet article

Martinot, C. (2013). La complexité d'un phénomène linguistique est-elle toujours source de difficulté ? Cas de l'acquisition des relatives en langue première (français et allemand). Nouvelles perspectives en sciences sociales, 9(1), 123-169. https://doi.org/10.7202/1024040ar

\section{Résumé de l'article}

Le système des relatives est considéré comme un système complexe. L'une des implications de cette affirmation devrait être que son acquisition réserve des difficultés aux enfants. À partir d'un protocole expérimental identique réalisé auprès d'enfants français de 4 à 10 ans et allemands de 6 à 10 ans qui consiste à demander à ces enfants de restituer l'histoire qui vient de leur être lue, individuellement, l'étude montre que sur les 6 relatives du texte source, seules 2 relatives explicatives, au poids diégétique important, ont été reformulées relativement souvent par une relative, et encore, seulement par les enfants français. L'occurrence d'un pronom relatif est cependant un critère insuffisant pour évaluer l'acquisition en cours du système des relatives. En revanche, la reformulation paraphrastique des phénomènes complexes attestés dans les relatives sources ( $v s$ effacement, changement de sens et répétition), fournit un critère fiable du degré d'acquisition des relatives. 


\title{
La complexité d'un phénomène linguistique est-elle toujours source de difficulté? Cas de l'acquisition des relatives en langue première (français et allemand)
}

\author{
Claire Martinot \\ Université Paris-Sorbonne, \\ EA $4509 \mathrm{STIH}$
}

\section{Les langues sont-elles des systèmes complexes?}

R ien qu'il n'existe pas, à notre connaissance, de définition satis-

0 faisante du concept de complexité qui puisse s'appliquer à l'ensemble des systèmes complexes (en physique, en biologie, dans les rapports sociaux...). Nous pouvons cependant caractériser les langues humaines comme des systèmes complexes d'après les critères suivants ${ }^{1}$.

1. Une langue fonctionne en tant que langue du fait des relations qui existent entre ses composants.

2. Ces composants sont reliés de telle façon que l'usager ne peut pas les séparer. La moindre production linguistique doit, en effet, satisfaire à une condition de complétude admise par toute la communauté qui pratique cette

Nous avons adapté à la réalité du fonctionnement des langues ce que Heylighen dit des systèmes complexes en général. Voir Francis Heylighen, "Five questions on complexity ", dans Carlos Gershenson (dir.), Complexity: 5 questions. Copenhagu, Automatic Press/VIP, 2008, en ligne : http://arxiv. org/ftp/nlin/papers/0702/0702016.pdf (consulté en août 2012). 
langue ${ }^{2}$. Par exemple, les enfants commencent à chanter et les enfants commencent. Nous désignerons cette production minimale complète et porteuse de sens par le terme de prédication. À la suite de Harris ${ }^{3}$, nous considérons qu'une langue est un ensemble de prédications ${ }^{4}$.

De la même façon, le descripteur de cette langue peut difficilement rendre compte du fonctionnement global de la langue s'il isole les niveaux phonétique, lexical, morphologique, syntaxique, sémantique et pragmatique.

3. Ces relations ne sont pas données objectivement et elles sont de nature différente selon qu'elles s'appliquent aux unités (par exemple, la relation entre le nom sujet et le verbe dans une prédication), aux sous-systèmes (système des relatives c. système plus global de la subordination) $)^{5}$, ou encore aux niveaux linguistiques.

4. Le fonctionnement et l'évolution d'une langue se caractérisent par de nombreuses distinctions et variations qui ont tendance à accroître le désordre du système, mais aussi par des règles qui concourent, au contraire, à maintenir un certain ordre. La langue est donc à michemin entre l'ordre et le désordre.

5. Une langue fonctionne du fait des locuteurs qui l'utilisent dans des environnements, eux-mêmes en interaction avec

2 Amr H. Ibrahim, "Les conditions de la prédication dans les langues », dans Amr H. Ibrahim (dir.), Prédicats, prédication et structures prédicatives, Paris, Cellule de Recherche en Linguistique, 2009, p. 12-49.

3 Zellig S. Harris, Language and Information, New York, Columbia University Press, 1988.

4 Ce point de vue sur la langue est compatible, et nous le revendiquons, avec le fait que ces prédications sont produites par des locuteurs particuliers dans des contextes particuliers.

5 Des chercheurs comme Miestamo donnent à " sous-système » un sens assez général, désignant par exemple l'ensemble des moyens dont dispose une langue pour exprimer la négation, l'interrogation... C'est à ce niveau relativement général du fonctionnement de la langue que nous faisons référence en parlant du sous-système des relatives. Voir Matti Miestamo, « Grammatical complexity in a cross-linguistic perspective ", dans Matti Miestamo, Kaius Sinnemäki et Fred Karlsson (dir.), Language Complexity: Typology, Contact, Change, Amsterdam/Philadelphia, Benjamins, 2008, p. 23-42. 
d'autres environnements. L'adaptation et l'auto-organisation qui caractérisent les systèmes dynamiques complexes sont donc nécessairement à l'œuvre dans la pratique langagière. Pensons à l'accent que l'on emprunte partiellement ou momentanément à son interlocuteur, à la réorganisation d'une information longue, impliquant de nombreux paramètres, en une information réduite à deux lignes écrites.

6. L'évolution à court ou à long terme d'une langue est imprévisible.

Ces critères généraux permettent de parler d'une complexité linguistique globale. Miestamo ${ }^{6}$ distingue cette complexité générale, partagée par toutes les langues, d'une complexité linguistique locale, liée à un sous-système de la langue. C'est de cette complexité locale dont nous parlerons ici à propos des relatives en français et en allemand. Cependant il ne s'agira pas de comparer les deux sous-systèmes pour savoir si l'un est plus complexe que l'autre selon un point de vue typologique, interne à la langue, mais d'analyser comment de jeunes locuteurs traitent les relatives. Nous rechercherons donc s'il y a une corrélation systématique entre un phénomène décrit par les linguistes comme complexe et la difficulté rencontrée par les jeunes locuteurs à produire des relatives dans le contexte d'une restitution d'histoire. Nous désignons par "difficulté » ce que d'autres appellent la " complexité d'usage "(agent-related complexity ${ }^{7}$ ). Nous pensons qu'il y a des degrés de complexité linguistique, comme il y a des degrés de difficulté pour les locuteurs. Nous reviendrons sur ces critères plus loin. Mais nous pouvons déjà dire que, dans la tâche expérimentale utilisée, la difficulté de restitution des relatives ne sera pas la même chez les enfants de 4 ans ou de 10 ans, elle sera éventuellement de nature différente chez les enfants francophones et germanophones, elle sera surtout déterminée par l'ensemble des caractéristiques linguistiques et discursives de chaque relative.

Matti Miestamo, op. cit.

Östen Dahl, The Growth and Maintenance of Linguistic Complexity, Amsterdam / Philadelphia, Benjamins, 2004. 
Nous détaillerons ces caractéristiques lors de l'analyse des reformulations de chaque relative. Les difficultés de production sont repérables en fonction des critères suivants ${ }^{8}$ :

1. suppression totale de la relative,

2. reprise très lacunaire de la relative de départ conduisant à un énoncé non permis par la langue,

3. changement de sens,

4. faible proportion de reformulations.

\section{Nouvelle hypothèse acquisitionnelle}

L'étude que nous présentons s'inscrit dans le cadre d'une nouvelle hypothèse acquisitionnelle ${ }^{9}$ qui propose une explication à la question, centrale en acquisition, de savoir comment les enfants passent des énoncés qu'ils entendent dans leur langue première aux énoncés qu'ils produisent eux-mêmes. Ce passage se réalise par un ensemble de transformations, désigné par le concept de reformulation, que l'enfant applique aux énoncés qu'il garde en mémoire. Cette hypothèse a été testée et confirmée par le projet international Acquisition et Reformulation (2003-2010) ${ }^{10}$. Le concept de reformulation est à entendre comme une sorte de négociation que fait le locuteur entre une partie invariante que l'enfant reprend à l'énoncé source et une

$8 \quad$ Cette étude se limite à l'analyse des productions linguistiques et n'abordera pas la question de la compréhension pour laquelle un protocole expérimental particulier serait nécessaire. Il est probable que, dans certains cas, la difficulté rencontrée par les enfants se situe au niveau de la compréhension. Cependant, le mode de reformulation analysé ici fournit un bon indice du degré de compréhension. La question de savoir si les enfants ont compris reste en suspens dans les cas où ils ne reformulent pas du tout mais aussi dans certains cas de répétition.

9 Claire Martinot, "Étude comparative des processus de reformulation chez des enfants de 5 à 11 ans ", Langages, n' 140, 2000, p. 92-123.

10 Le projet Acquisition et Reformulation, dont certaines publications se trouvent dans les références bibliographiques de cet article, a porté et porte encore sur les langues premières suivantes : français, italien, roumain, croate, polonais, allemand, arabe yéménite. La comparaison français-allemand a fait l'objet d'une étude portant sur l'acquisition des prédications secondes (Claire Martinot et coll., "Prédication principale vs seconde à l'épreuve des faits d'acquisition ", dans Amr H.Ibrahim (dir.), Prédicats, prédication et structures prédicatives, Paris, Cellule de Recherche en Linguistique, 2009, p. 50-81). 
partie inédite. Ce qui change comme ce qui est maintenu peut se situer au niveau lexical, syntaxique ou sémantique ${ }^{11}$.

Au cours de la reformulation d'un énoncé source, l'enfant réalise l'une des trois postures suivantes : 1) il répète l'énoncé source à l'identique ou presque; 2) il modifie l'énoncé source en donnant un autre sens à l'énoncé reformulé (tout en maintenant un invariant formel : le lexique ou la construction); 3) il modifie (formellement) l'énoncé source tout en conservant le sens de celui-ci.

L'ensemble des résultats issus du projet Acquisition et Reformulation montre la même tendance au même âge quelle que soit la langue première : les reformulations par répétition sont beaucoup plus fréquentes que les autres postures de reformulation chez les enfants les plus jeunes (bien qu'il s'agisse des enfants de 4 ans dans le projet Acquisition et reformulation, cette posture apparaît bien avant); les reformulations impliquant un changement de sens sont très fréquentes à 6 et 8 ans, et les reformulations par équivalence sémantique sont les plus fréquentes chez les enfants de 10 ans. Ces résultats nous permettent donc de faire l'hypothèse que les reformulations par équivalence sémantique sont plus difficiles à réaliser que celles qui entraînent un changement de sens ou que les répétitions. De plus, nous avons pu établir que les différents types de paraphrases ne sont pas les mêmes, tendanciellement, selon l'âge ${ }^{12}$, ce qui nous permet de tirer la même conclusion que précédemment : certaines paraphrases sont plus difficiles à produire que d'autres. Dans l'ordre croissant de difficulté, on trouve d'abord les paraphrases descriptives définitoires, dans lesquelles un mot de l'énoncé source est reformulé par une courte séquence définitoire, puis les paraphrases sémantiques dans lesquelles un mot de l'énoncé source

\footnotetext{
11 Claire Martinot, La reformulation dans des productions orales de définitions et explications. (Enfants de maternelle), Thèse de Doctorat, Université Paris VIII, 1994.

12 Claire Martinot, "Reformulations paraphrastiques et stades d'acquisition en français langue maternelle ", Cahiers de praxématique, $\mathrm{n}^{\circ}$ 52, 2009, p. 29-57; Claire Martinot, «Reformulation et acquisition de la complexité linguistique ", Travaux de linguistique, n 61, 2010, p. 63-96.
} 
est reformulé par un synonyme, puis les paraphrases formelles dans lesquelles l'ensemble de l'énoncé source est transformé (passif > actif, par exemple) ou restructuré (le verbe, par exemple, est construit différemment tout en gardant le même sens) et, enfin, les paraphrases logiques dans lesquelles l'équivalence n'est attestée qu'en discours ${ }^{13}$. Ces tendances sont indicatives et doivent, bien entendu, être modulées en fonction de la complexité linguistique de l'énoncé source, d'une part, et en fonction du nombre d'énoncés équivalents disponibles dans la langue, d'autre part. On remarquera que si un terme a dix synonymes dans une langue donnée, ce fait interne à la langue entraîne une plus grande complexité locale que dans le cas où un terme n'a pas de synonyme, mais la difficulté pour l'usager, dans une tâche de restitution, est inversée : si un terme n'a pas de synonyme, le locuteur est contraint de se souvenir de ce seul terme, il n'a pas d'autre alternative.

\section{Protocole expérimental}

Pour pouvoir observer systématiquement, et à partir d'un même texte source, comment les enfants reformulent un phénomène donné par la recherche comme complexe, nous utilisons un protocole semi-expérimental qui consiste à lire à chaque enfant l'histoire de Tom et Julie/Tom und Julia (voir annexe) et à lui demander de raconter à son tour l'histoire " avec ses mots à lui, sans rien oublier ". Chaque histoire ainsi restituée fournit l'ensemble des énoncés reformulés que nous pouvons alors comparer avec les énoncés sources correspondants. L'analyse du différentiel entre chaque paire, constituée d'un énoncé source et d'un énoncé reformulé, requiert un découpage rigoureux en prédications

13 Nous avons d'abord désigné (Claire Martinot, 2009, 2010, op. cit.) les paraphrases logiques par "paraphrases fonctionnelles ». Depuis, et dans cette livraison, nous optons pour " paraphrases logiques " qui est plus transparent que " paraphrases fonctionnelles » et qui réfere à des paraphrases dans lesquelles les locuteurs interprètent l'énoncé source en fournissant selon les cas une cause, une conséquence, une explication, souvent corrélées à un nouveau point de vue. Voir Claire Martinot, "La reformulation : de la construction du sens à la construction des apprentissages ", (sous presse a : http://www.revue-corela.org). 
élémentaires, seules unités en langue qui permettent de distinguer une répétition plus ou moins stricte, d'une paraphrase d'un type particulier ou d'un changement de sens. Par ailleurs, on considère que la restitution d'un texte est un condensé temporel et linguistique de la situation naturelle d'acquisition du fait que le texte source est plus complexe ${ }^{14}$ que le texte reformulé, et que les enfants sont " obligés " de puiser dans ce texte pour raconter à leur tour l'histoire, tout comme ils le font en situations naturelles lorsqu'ils puisent dans les discours de leur entourage proche et dans les textes qui leur sont lus ou racontés.

Les six relatives des textes sources français et allemand seront analysées du point de vue de la reformulation constatée, c'est-àdire de la tendance différentielle la plus marquée pour une prédication donnée ${ }^{15}$ : L'énoncé reformulé sera ainsi étiqueté comme une répétition, une reformulation avec changement de sens, ou une équivalence sémantique, correspondant aux différents types de paraphrases.

\section{Complexité linguistique des relatives et difficulté de la tâche de reformulation}

Nous rassemblons ci-dessous les raisons qui fondent les descripteurs des langues à considérer les relatives comme des configurations complexes.

1. La relative est rattachée à un nom antécédent, elle complexifie donc ce nom même dans le cas où elle n'est pas incise : Par exemple, j'ai vu le voisin qui vient d'emménager - j'ai vu le voisin qui vient d'emménager sortir de la boulangerie. La relation sémantique a ici lieu entre un nom et une

14 L'histoire lue aux enfants a été rédigée dans un style proche de la norme académique, avec une haute densité informative, de nombreuses prédications à deux prédicats (les fleurs se parlent en chantant), de nombreux compléments antéposés au verbe..., toutes caractéristiques absentes de la langue orale adressée en général aux enfants.

15 En effet, dans toute prédication reformulée, on peut trouver un ou deux mots répétés, mais aussi un mot qui va introduire un léger changement de sens. Pourtant la tendance globale du différentiel est en général nette : tendance orientée vers la répétition, vers le changement de sens ou vers l'équivalence informationnelle. 
proposition et non entre deux propositions comme c'est le cas général dans les subordonnées.

2. La relation sémantique entre l'antécédent et la relative explicative ou déterminative n'est pas marquée alors qu'elle est de nature différente. La relative explicative apporte une information nouvelle à la suite d'une prédication première exprimée dans la principale; la relative déterminative sert à identifier l'antécédent. D'après nos résultats ${ }^{16}$, les relatives déterminatives semblent être plus difficiles à acquérir que les relatives explicatives ${ }^{17}$.

3. Le pronom relatif a une triple fonction : (1) pronom anaphorique du nom antécédent, (2) connecteur entre la principale et la relative, (3) sujet ou complément du verbe de la relative.

Mais il est évident que la complexité attachée aux relatives ne se réduit pas aux phénomènes ci-dessus : une relative peut être encore plus complexe si, par exemple, elle contient une métaphore ([...] la lumière qui inondait l'intérieur de l'arbre), si elle contient un verbe opérateur dont le sens n'est pas accessible aux jeunes enfants (où les fleurs semblaient se parler) et que, de surcroît, ce verbe s'applique à une prédication complexe (où les fleurs semblaient se parler en chantant).

16 Claire Martinot et coll., 2009, op. cit.

17 De nombreux travaux ont analysé l'acquisition des relatives, (voir Lisa Dasinger et Cecile Toupin, "The Development of Relative Clause Function in Narrative ", dans Ruth A. Berman et Dan I. Slobin (dir.), Relating Events in Narrative. A Crosslinguistic Developmental Study, Hillsdale, New Jersey, Lawrence Erlbaum, 1994, p. 457-515; Holger Diessel, The Acquisition of Complex Sentences, Cambridge, Cambridge University Press, 2004), mais la distinction n'est pas évoquée entre les explicatives et les déterminatives de même que la distinction entre les "fausses " relatives de type il y a le facteur qui sonne et les vraies relatives de type le facteur qui a apporté le courrier hier est très gentil (relative déterminative) ou hier le facteur m'a apporté le courrier qui était destiné à ma voisine (relative explicative si l'on marque une pause avant qui, on pourra en conclure que le facteur s'est trompé; ou relative déterminative si l'on ne marque pas de pause avant qui, on pourra en conclure que le facteur ne m'a apporté que ce courrier, il est venu exprès). Pour une revue éclairante de ces distinctions, voir Georges Kleiber, "Relatives restrictives / relatives appositives : dépassement(s) autorisé(s) ", Langages, nº 88, 1987, p. 41-43. 
Nous verrons donc comment les enfants francophones et germanophones reformulent les différentes relatives. Cette analyse permettra de montrer que les locuteurs rencontrent de moins en moins de difficultés à reformuler les relatives en fonction de leur âge. Par conséquent, le nombre absolu de reformulations augmentera de même que la proportion des reformulations paraphrastiques. De façon corollaire, les répétitions et les changements de sens diminuent jusqu'à disparaître à 10 ans. Parmi les reformulations paraphrastiques, les paraphrases formelles (transformations et restructurations) et les paraphrases logiques sont majoritaires à 10 ans. Du point de vue de la tâche de reformulation, elles sont difficiles à mettre en œuvre parce que les premières conservent les mêmes mots lexicaux dans une autre configuration syntaxique, tandis que les secondes disent tout autre chose en établissant cependant une relation sémantique avec l'énoncé source sans que la suite du texte en soit affectée.

Nous analyserons successivement les six relatives dans l'ordre des séquences de l'histoire en fournissant à chaque fois des remarques générales sur les raisons (supposées) de la complexité particulière de chacune des relatives, sur son statut sémantique (relative explicative ou déterminative), sur son statut informationnel par rapport au fil narratif. Nous analyserons ensuite les procédures de reformulation en les présentant dans un ordre croissant de difficulté ${ }^{18}$.

\section{Reformulations de la relative de la séquence 1}

[Fr.] Elle tenait par la main une petite fille que personne n'avait encore jamais vue.

[All.] Sie hatte ein kleines Mädchen an der Hand, das noch nie jemand gesehen hatte.

18 Il n'est pas toujours aisé de savoir si, par exemple, les reformulations par répétitions sont plus ou moins difficiles à produire que les reformulations entraînant un changement de sens. En revanche, ces deux types de reformulation sont toujours plus souvent attestés chez les plus jeunes enfants. De même, les reformulations par paraphrase logique ou formelle sont plus difficiles à réaliser que les autres types de reformulations, mais leur degré de difficulté relatif est, selon les cas, permutable. 


\section{Remarques générales}

La relative de la séquence 1 est une relative explicative apportant une information pertinente dans la narration : d'une part, cette information correspond à un événement pour les enfants scolarisés, d'autre part, elle peut expliquer le retard inhabituel de l'enseignante (voir la phrase précédente dans l'annexe). Du point de vue grammatical, elle ne présente pas de complexité particulière; du point de vue sémantique, l'interprétation de personne dans une phrase négative peut présenter une difficulté pour les enfants les plus jeunes.

Procédures de reformulation

La relative source est de plus en plus souvent reformulée, quelle que soit la forme de la reformulation, avec cependant un infléchissement chez les 10 ans dans les deux langues.

En allemand comme en français, deux procédures de reformulation sont caractéristiques : la tendance à répéter chez les plus jeunes et la reformulation par paraphrase logique chez les plus âgés. Ainsi de nombreux enfants, dans les deux langues, ont reformulé l'information exprimée par la relative source en proposant une cause : personne n'avait jamais vu la fillette parce qu'elle fait sa rentrée à l'école, ou vient pour la première fois, ou entre dans une nouvelle école. Hors contexte, ces reformulations auraient un sens différent relativement à personne n'avait encore jamais vue cette petite fille. De plus, ces reformulations par paraphrase logique introduisent un nouveau point de vue, c'està-dire, ici, une nouvelle relation actantielle, puisque la petite fille devient le sujet du procès. 


\section{Reformulations par répétition}

Adrien ( 4 ans) : (...) *elle tenait une petite fille que tout le monde n'avait jamais vue ${ }^{19}$.

Achille (6 ans) : (...) elle tenait dans sa main une petite fille qu'on n'avait encore jamais vue.

Emily (8 ans) : (...) sie hatte eine Schülerin, * den *den niemand bisher gesehen hatte $e^{20}$ [elle avait une élève que personne n'avait vue jusqu'à présent. Le pronom relatif den est au masculin au lieu d'être au féminin].

Sebastian (10 ans) : und hatte so ein kleines Mädchen an der Hand gehabt, und das hat noch nie jemand vorher gesehen da [et avait eu une petite fille à la main, et encore personne ne l'avait jamais vue avant].

Reformulations par paraphrase sémantique

Les paraphrases sémantiques expriment une équivalence sémantique, quel que soit le contexte et sans changer le point de vue, y compris quand toute la proposition relative du texte source est reformulée par un seul adjectif, comme c'est le cas chez les enfants germanophones (un petit tiers à 8 et 10 ans) qui ont réduit la relative en un adjectif neue/nouvelle. Quelques reformulations par paraphrase sémantique sont attestées dès 6 ans (fr.) :

Sacha (6 ans) : ce matin la maitresse elle tenait la main à une nouvelle élève.

Hanna (10 ans) : sie hatte an der Hand *ein ganz neue Mitschülerin [elle avait à la main une toute nouvelle élève. Larticle indéfini ein est au neutre au lieu d'être au féminin].

Natalie (10 ans) : die hatte ein Kind, die neue Schülerin [elle avait un enfant, la nouvelle élève].

19 L'astérisque signale que l'énoncé qui suit le symbole n'est pas prévu par la langue, quelle que soit la situation d'énonciation ou la norme de référence. On dira qu'il est agrammatical.

20 Les reformulations des enfants germanophones sont traduites de façon non littérale pour en faciliter la compréhension. 
Chez les enfants francophones de 8 ans, les paraphrases sémantiques prennent des formes variées (connaître, élèves):

Rémy (8 ans) : elle tenait la main d'une petite fille qu'on ne connaissait pas.

Ninon (8 ans) : elle tenait par la main un enfant que les élèves n’avaient encore jamais vu.

\section{Reformulations par paraphrase logique}

Mona (6 ans) : da ist eine neue Schülerin *herkommen [alors une nouvelle élève est venue. Le participe passé herkommen (forme infinitive) devait avoir la forme suivante hergekommen].

Hanna (8 ans) : da kommt eine neue Mitschülerin [alors une nouvelle élève vient].

Dominik (8 ans) : da ist die Julia in eine neue Klasse gekommen [alors Julia est venue dans une nouvelle classe].

Pamela (10 ans) : (...) mit einem Kind an der Hand, das noch nie an dieser Schule war [avec un enfant à la main qui n'avait encore jamais été dans cette école].

Maurine (10 ans) : c'est l'histoire d'une petite fille qui vient pour la première fois dans une école.

Théo (10 ans) : c'est Julie qui entre dans une nouvelle école.

Maxime (10 ans) : il était une fois une petite fille qui faisait sa rentrée à l'école. 
Tableau 1 : Répartition des types de reformulation de la relative source dans la séquence 1

\begin{tabular}{|c|c|c|c|c|c|c|c|}
\hline & \multirow{2}{*}{$\begin{array}{c}4 \text { ans } \\
\text { Fr. } \\
\mathrm{N}=15\end{array}$} & \multicolumn{2}{|c|}{6 ans } & \multicolumn{2}{|c|}{8 ans } & \multicolumn{2}{|c|}{10 ans } \\
\hline & & $\begin{array}{c}\text { Fr. } \\
N=15\end{array}$ & $\begin{array}{c}\text { All. } \\
\mathrm{N}=15\end{array}$ & $\begin{array}{c}\mathrm{Fr} . \\
\mathrm{N}=14\end{array}$ & $\begin{array}{c}\text { All. } \\
N=16\end{array}$ & $\begin{array}{l}\text { Fr. } \\
N=15\end{array}$ & $\begin{array}{l}\text { All. } \\
\mathrm{N}=15\end{array}$ \\
\hline Répétitions & $3 / 3$ & $5 / 7$ & & $1 / 8$ & $3 / 8$ & & $1 / 6$ \\
\hline $\begin{array}{l}\text { Reformulations avec changement } \\
\text { de sens }\end{array}$ & & & & & $1 / 8$ & & \\
\hline Paraphrases sémantiques & & $2 / 7$ & & $3 / 8$ & & $2 / 7$ & $2 / 6$ \\
\hline Paraphrases logiques & & & $2 / 2$ & $4 / 8$ & $4 / 8$ & $5 / 7$ & $3 / 6$ \\
\hline $\begin{array}{l}\text { TOTAL des reformulations de la } \\
\text { relative / reformulations de la } \\
\text { séquence }\end{array}$ & $3 / 15$ & $7 / 15$ & $2 / 15$ & $8 / 14$ & $8 / 16$ & $7 / 15$ & $6 / 15$ \\
\hline $\begin{array}{l}\text { Occurrences d'une relative } \\
\text { (reformulation de la relative } \\
\text { source) }\end{array}$ & $3 / 3$ & $5 / 7$ & & $3 / 8$ & $3 / 9$ & $1 / 7$ & $1 / 6$ \\
\hline $\begin{array}{l}\text { Introduction d'une nouvelle } \\
\text { relative dans la séquence }\end{array}$ & & & & $7 / 14$ & & $7 / 15$ & \\
\hline
\end{tabular}

\section{Conclusion}

Les procédures de reformulations font apparaître trois tendances.

1. La grande différence quantitative, constatée à 6 ans entre les enfants des deux langues ( 2 enfants germanophones reformulent la relative contre 7 en français) disparaît ensuite.

2. La réduction de la relative par un adjectif est beaucoup plus fréquente en allemand qu'elle ne l'est en français.

3. L'introduction de "nouvelles relatives " n'est attestée qu'en français, il s'agit soit du dispositif extrêmement productif dans cette langue de c'est... qui ou il y a... qui ou il était une fois... qui, soit de relatives qui transfèrent dans la séquence 1 une information de la séquence 2 : qui s'appelle Julie. 
Concernant la relation entre la complexité supposée de cette relative et la difficulté de la tâche de restitution, on peut confirmer que l'enchaînement : Elle tenait par la main une petite fille que personne n'avait encore jamais vue, n'a pas posé de problème de compréhension aux enfants qui ont reformule l'ensemble puisqu'aucun changement de sens n'est attesté. Au niveau de la production, la reformulation de la relative peut être considérée comme difficile chez les plus jeunes puisque seulement trois enfants francophones de 4 ans sur 15 et seulement deux enfants germanophones de 6 ans sur 15 ont restitué la relative (répétition plus ou moins réussie).

À 6 ans (fr.), la répétition se généralise sans erreur et quelques enfants réduisent la relative à un adjectif épithète, comme le font les enfants germanophones à tous les âges. Cette dernière procédure peut être considérée comme une reformulation simplificatrice, plus ou moins productive selon la langue.

L'augmentation des paraphrases sémantiques avec reprise du relatif chez les francophones de 8 ans permet de dire que cette construction est maîtrisée à cet âge. Un phénomène comparable (paraphrase logique avec reprise du relatif) est attesté chez une fillette germanophone de 10 ans. La combinaison des deux critères (paraphrase + pronom relatif) permet de considérer ces reformulations comme des procédures complexes.

Les paraphrases logiques des germanophones (8 ans) avec changement de point de vue et réduction de la relative à un adjectif, d'une part, et, d'autre part, les « nouvelles relatives » des francophones (8 ans) montrent qu'à 8 ans, les tendances de la langue première - localisées dans la séquence étudiée - sont maîtrisées et permettent aux enfants un mode de restitution alternatif par rapport à la construction relative proposée dans le texte source. 


\section{Reformulations de la relative de la séquence 4}

[Fr.] il s'est dirigé vers la fillette et lui a tendu la boîte qu'il avait fabriquée pour elle la veille.

[All.] ging er auf sie zu, und hielt ihr die Schachtel hin, die er am Vortag für sie gebastelt hatte.

Remarques générales

La relative de cette séquence est une relative déterminative, que nous supposons plus complexe qu'une relative explicative. En effet, l'information apportée à l'antécédent est indispensable pour déterminer de quelle entité on parle. Mais, dans le cas qui nous occupe ici, cette information est redondante du fait qu'il n'y a pas d'autre boîte dans l'histoire, et que l'on a appris dans la séquence précédente que Tom a fabriqué chez lui une boîte pour Julie. Il n'est donc pas étonnant que la quasi-totalité des enfants supprime cette non-information : un seul francophone de 8 ans l'a reformulée, par une relative (paraphrase sémantique lacunaire) :

Claire (8 ans) : il lui donna une jolie (\#) boule en en en dorée qu'il avait faite chez lui ${ }^{21}$.

\section{Reformulations de la relative de la séquence 6}

[Fr.] Tom souleva le couvercle et découvrit un morceau de papier sur lequel Julie avait écrit (...).

[All.] Tom hob den Deckel ab, und entdeckte ein Stück Papier, auf das Julia geschrieben hatte.

Remarques générales

La relative explicative de cette séquence ne présente pas de difficulté particulière au niveau sémantique. Au niveau syntaxique,

$\overline{21}$ Le symbole \# note un changement de sens. Ici, ce changement n'affecte que la boite ronde qui est devenue une boule. L'information globale fournie par l'enfant peut tout de même être considérée comme une équivalence informationnelle. 
on peut remarquer que le sujet de la relative Julie est différent du sujet de la principale, Tom, et que la question " qui fait quoi " posera peut-être quelques difficultés aux enfants. Au niveau narratif, la séquence 6 dans son entiereté est très importante puisqu'elle marque le passage entre l'univers familier de la classe et celui, magique, dans lequel Julie invite Tom. Cette séquence a été la plus reformulée, à tous les âges et dans les deux langues (de même que la séquence 13, tableau 6). Toujours au niveau de la pertinence narrative, le contenu sémantique de la relative est nécessaire pour introduire l'objet de l'invitation à aller le soir dans la forêt.

Procédures de reformulation

Dans les deux langues, la procédure de reformulation que nous avons étiquetée "reformulation par équivalence sémantique lacunaire " (sous-catégorie des paraphrases sémantiques) est de loin la plus fréquente à tous les âges. Elle est lacunaire parce que le sujet Julie /Julia du verbe relatif est effacé, transformant l'énoncé source de la forme personnelle à la forme impersonnelle. Nous considérons tout de même que, malgré l'absence de cette information, on a une équivalence sémantique parce que le contenu du message permet de déduire que c'est bien Julie qui l'a écrit (ex., retrouve-moi à 8 h sous l'arbre grand). Les paraphrases logiques introduisent ici une relation de conséquence entre le verbe source : Julie avait écrit et le verbe reformulé : Tom lit. De façon surprenante, elles sont attestées dès 4 ans chez les francophones et à 8 ans chez les germanophones. Les restructurations par réduction de la relative en français (par exemple, il trouva un petit papier écrit) et par l'emploi d'une construction directe du verbe écrire en allemand et en français sont également attestées plus tôt chez les francophones (6 ans c. 8 ans chez les germanophones). Les paraphrases logiques manifestent une bonne compréhension de l'histoire sans être syntaxiquement difficiles à produire, tandis que les restructurations doivent être considérées comme des procédures difficiles à réussir puisque le même lexique est construit d'une façon nouvelle. La réutilisation du 
pronom relatif est très minoritaire en allemand; en français, elle augmente entre 4 et 8-10 ans. Ces dernières tranches d'âge voient les reformulations avec relatif atteindre la moitié des énoncés reformulés.

Reformulations par répétition

De façon générale, la répétition ou quasi-répétition (avec changement du temps verbal par exemple) est peu présente. Nous relevons quelques attestations en allemand, mais aucune en français :

Julian (6 ans) : da hat sie drauf geschrieben [alors elle a écrit dessus].

Thomas (8 ans) : worauf Julia geschrieben hat [sur lequel Julia a écrit].

Florian (10 ans) : wo sie draufgeschrieben hatte [où elle avait écrit dessus/ sur lequel elle avait écrit].

Reformulations par paraphrase sémantique lacunaire

Les reformulations par équivalence sémantique lacunaire correspondent en français à deux constructions majoritaires : c'était écrit/marqué et il y avait écrit/marqué. En allemand, la forme impersonnelle est construite avec un autre verbe darauf stehen [adverbe locatif - V de position, l'ensemble est équivalent à il y a]. Chez les germanophones de 10 ans, cette construction impersonnelle est très fréquente. Dans les deux langues, ce type de reformulation simplifie l'énoncé source, d'autant plus quand la construction relative est supprimée (Solène, Mona, Sophie, Natalie) :

Laurana ( 4 ans) : (enlève le couvercle de la boîte et) où c'était écrit (...).

Marion (4 ans) : il y avait un petit papier sur lequel il y avait écrit.

Solène ( 8 ans) : et sur ce bout de papier il est écrit.

Barbara (10 ans) : et trouva un petit papier où il y avait marqué.

Mona (6 ans) : da hat er ein Papier gesehen, da stand was drauf (...) [alors il a vu un papier, alors il y avait quelque chose dessus...].

Sophie (8 ans) : hat drin einen Zettel gefunden (...), da stand d(a) rauf(...) [il a trouvé dedans un papier, alors il y avait dessus...]. 
Natalie (10 ans) : und dann hat er sie geöffnet und dann stand auf einem Zettel [et après il l'a ouverte et après il y avait sur un papier ...].

Reformulations par paraphrase sémantique complète

Les paraphrases sémantiques complètes sont rares, seulement attestées en français où les enfants ont utilisé des verbes synonymes, plus fréquents à l'oral que écrire (un enfant de 8 ans et deux enfants de 10 ans) :

Océane ( 8 ans) : après Julie elle fait un mot à Tom.

Marion X (10 ans) : Julie a marqué dans sa boîte un petit mot à Tom.

Reformulations par paraphrase logique : Julie écrit > Tom lit

Bien qu'elles soient attestées dès 4 ans (1 enfant francophone), elles sont peu fréquentes en français, mais elles augmentent légèrement à 10 ans. Chez les germanophones, seuls 2 enfants de 8 ans l'utilisent.

Clara ( 4 ans) : Tom lit un petit mot dans la boîte il lit le petit mot et c'était de la retrouver ce soir (...).

Madeleine (6 ans) : Tom ouvrit la boîte et vit un papier qu'il lit.

Maurine (10 ans) : il ouvrit le couvercle et * lit attentivement le mot qui était dedans (...).

Mona (8 ans) : hat der Tom dann gelesen [après Tom a lu].

Reformulations par paraphrase formelle (restructuration)

Un premier type de restructuration, attesté dans les deux langues, correspond aux reformulations dans lesquelles le verbe schreiben/ écrire est construit avec un complément direct (à l'accusatif en allemand) au lieu d'un complément prépositionnel comme dans le texte source auf das acc. $/$ sur lequel. : auf das Julia geschrieben hatte [sur lequel Julia avait écrit]).

Emily (8 ans) : die Julia hat ihn geschrieben [Julia l'a écrit].

Anja (8 ans): und dann hat sie einen Zettel geschrieben [et après elle a écrit un papier].

Sacha (6 ans) : il y avait un petit papier que Julie avait raconté avait écrit. 
Un deuxième type de restructuration est attesté chez les francophones seulement. Il s'agit de restructuration par réduction de la relative (l'actant sujet est alors effacé) :

Margot (6 ans) : Tom ouvrit la boîte et *vu un mot écrit.

Romain (10 ans) : Tom ouvrit et vit écrit sur le papier.

Camille (10 ans) : il trouva un petit papier écrit.

Un troisième type de restructuration qui a entraîné de nombreux changements morphosyntaxiques est attesté chez une seule fillette germanophone de 10 ans :

Jennifer (10 ans) : wo drauf stand in Julias Schrift (...) [sur lequel il y avait avec l'écriture de Julia...].

Reprise de la relative en français c. adverbe anaphorique en allemand La reformulation de la relative source par une relative augmente nettement en français ( 4 occurrences à 4 ans, 7 à 8 et 10 ans). En allemand, la formulation d'une relative reste très marginale. Les enfants germanophones reformulent majoritairement, à tous les âges, la relative source par une proposition non subordonnée, introduite par un adverbe anaphorique : da [là], darauf: advprép. équivalent à sur cela], qui reprend ein Stück Papier [un morceau (de) papier] de la proposition précédente.

Kimberly (6 ans) : und da war ein kleiner Zettel drin, da steht (...) [et là était un petit papier dedans, il y a là (...)].

Simone (8 ans) : und sah einen Zettel drin, darauf stand (...) [et vit dedans un papier, il y avait dessus...] 
Tableau 2 : Répartition des types de reformulation de la relative dans la séquence 6

\begin{tabular}{|c|c|c|c|c|c|c|c|}
\hline & 4 ans & $6 a$ & ans & $8 a$ & & 10 & ans \\
\hline & $\begin{array}{c}\text { Fr. } \\
\mathrm{N}=12\end{array}$ & $\begin{array}{c}\text { Fr. } \\
\mathrm{N}=15\end{array}$ & $\begin{array}{c}\text { All. } \\
\mathrm{N}=10\end{array}$ & $\begin{array}{c}\mathrm{Fr} . \\
\mathrm{N}=15\end{array}$ & $\begin{array}{c}\text { All. } \\
\mathrm{N}=13\end{array}$ & $\begin{array}{c}\text { Fr. } \\
\mathrm{N}=15\end{array}$ & $\begin{array}{c}\text { All. } \\
\mathrm{N}=15\end{array}$ \\
\hline Répétitions & & & $2 / 9$ & & $3 / 13$ & & $1 / 13$ \\
\hline $\begin{array}{l}\text { Reformulations avec } \\
\text { changement de sens }\end{array}$ & $1 / 11$ & $1 / 11$ & & & & & \\
\hline $\begin{array}{l}\text { Paraphrases } \\
\text { sémantiques lacunaires }\end{array}$ & $9 / 11$ & $4 / 11$ & $7 / 9$ & $9 / 14$ & $5 / 13$ & $7 / 15$ & $10 / 13$ \\
\hline $\begin{array}{l}\text { Paraphrases } \\
\text { sémantiques complètes }\end{array}$ & & & & $1 / 14$ & & $2 / 15$ & \\
\hline Paraphrases logiques & $1 / 11$ & $3 / 11$ & & $3 / 14$ & $2 / 13$ & $4 / 15$ & \\
\hline Restructurations & & $3 / 11$ & & $1 / 14$ & $3 / 13$ & $2 / 15$ & $2 / 13$ \\
\hline $\begin{array}{l}\text { TOTAL des } \\
\text { reformulations de la } \\
\text { relative / reformulations } \\
\text { de la séquence }\end{array}$ & $11 / 12$ & $11 / 15$ & $9 / 10$ & $14 / 15$ & $13 / 13$ & $15 / 15$ & $13 / 13$ \\
\hline Occurrence d'une relative & $4 / 11$ & $5 / 11$ & $1 / 9$ & $7 / 14$ & $1 / 13$ & $7 / 15$ & $2 / 13$ \\
\hline
\end{tabular}

\section{Conclusion}

Si dans les deux langues, la reformulation de la relative source par une paraphrase sémantique lacunaire est la procédure la plus fréquente à tous les âges, de nouvelles procédures de reformulation plus complexes sont attestées à partir de 8 ans dans les deux langues : il s'agit des reformulations paraphrastiques complètes (français seulement), des reformulations logiques (difficulté sémantique) et des reformulations par restructuration. 
Il est intéressant de noter l'attitude reformulatoire des enfants de 6 ou 8 ans, selon les langues. Ces enfants font preuve d'un grand souci de restituer le mieux possible l'histoire, alors qu'à 10 ans, malgré quelques restructurations très complexes (par exemple, Jennifer, en allemand), les enfants de cette tranche d'âge manifestent une plus grande uniformisation, probablement due à la scolarisation.

Nous pouvons constater la préférence du français pour les relatives contrairement à l'allemand qui préfère utiliser des adverbes anaphoriques ( $d a$, darauf) servant de connecteurs avec la proposition précédente.

\section{Reformulations de la relative de la séquence 11}

[Fr.] les enfants furent éblouis par la lumière qui inondait l'intérieur de l'arbre.

[All.] die Kinder wurden von dem Licht geblendet, das aus dem Inneren des Baumes kam.

Remarques générales

La relative source de la séquence 11 est complexe pour plusieurs raisons.

\section{La métaphore}

En français, mais pas en allemand, la relative comporte une métaphore. Une métaphore est linguistiquement complexe parce qu'elle résulte d'une comparaison non explicite entre, ici, le sujet de la relative la lumière et un sujet de type élément liquide plus fréquemment utilisé avec le verbe inonder. La tâche de reformulation d'un énoncé contenant une métaphore est également difficile parce qu'une métaphore n'est paraphrasable que si le locuteur, l'ayant comprise, en explique le mécanisme. Les enfants germanophones devraient donc avoir moins de difficultés que les enfants francophones lors de la restitution de la séquence 11. 


\section{Relative déterminative}

Le statut de relative déterminative ou restrictive implique que la portée de l'antécédent soit restreinte : il s'agit d'une lumière particulière et non de la lumière en général.

\section{Statut de l'information}

Le contenu sémantique de la relative n'a pas d'incidence sur la suite de l'histoire. Il est donc peu pertinent du point de vue narratif, ce qui constitue une difficulté supplémentaire de mémorisation.

Procédures de reformulation

La relative de cette séquence n'a été reformulée que par trois enfants francophones de 10 ans, tandis que les germanophones l'ont reformulée, certes peu souvent, mais dès 6 ans. Les procédures sont diversifiées et différentes d'une langue à l'autre. Les enfants germanophones ont produit des paraphrases logiques d'un type syntaxique particulier : ils ont synthétisé en une seule prédication une information issue de la principale (das Lichtlla lumière, strablen/rayonner) et une information issue de la relative (der Baumll'arbre, das Innerell'intérieur (de l'arbre), aus... kommen lsortir de).

Reformulations par répétition lacunaire

Cette procédure n'est attestée qu'en allemand du fait que la particule (he)raus, ne faisant pas partie du sens du verbe comme c'est le cas en français avec le verbe sortir, a été supprimée par Anja et Tamara. Thomas a supprimé la provenance de la lumière : Anja (8 ans) : und dann kam ein Licht [et après une lumière venait].

Tamara (8 ans) : und dann ein Licht ist gekommen [et après une lumière est venue].

Thomas (8 ans): dann kam ein Licht heraus [après une lumière sortait]. 


\section{Reformulations par répétition quasi complète}

Un seul cas est attesté en allemand :

Lukas (10 ans): sie wurden von einem Licht geblendet, das aus dem Baum raus kam [ils furent aveuglés par une lumière qui sortait de l'arbre].

Reformulation par paraphrase sémantique

Un seul cas est attesté chez un enfant allemand qui a fourni un synonyme du verbe de la relative :

Florian (10 ans) : dann ist ein Licht herausgeschienen [après une lumière a brillé en sortant].

Reformulations par paraphrase logique (déduction)

Chez les germanophones, ce type de reformulation est attesté aux trois âges, même s'il n'y a qu'une ou deux occurrences par tranche d'âge :

Daniel (6 ans) : da war ein gelbes Licht [information déduite de la principale] im Baumstamm [information provenant de la relative source] - [il y avait une lumière jaune dans le tronc d'arbre].

Chez les deux enfants suivants (Lukas et Cornelia), l'arbrelder Baum ou l'intérieur de l'arbreldas Innere des Baumes produit de la lumière/Licht:

Lukas (8 ans) : und dann hat der Baum so ein ganz helles Licht gemacht [et après l'arbre a fait/produit une lumière très blanche].

Cornelia (8 ans) : das Innere [information provenant de la relative] strablt [information provenant de la principale] - [l'intérieur rayonne].

Jennifer (10 ans) : raus kam [information provenant de la relative] ein ganz grosses Licht, also ganz ganz viel Licht [information provenant de la principale] - [une très grande lumière, oui vraiment beaucoup beaucoup de lumière sortait de là].

Anna-Maria (10 ans) : ist dann so ein helles Licht [information provenant de la principale] rausgekommen [information provenant de la relative] - [après une sorte de lumière blanche est sortie].

Chez Paul, on peut également considérer que sa reformulation paraphrastique provient d'une déduction : la quantité de lumière (inondait) a pour conséquence qu'elle déborde, qu’elle sort. En 
revanche, il n'y a pas de synthèse entre la principale et la relative, comme c'est le cas chez tous les enfants germanophones qui ont produit une reformulation logique :

Paul (10 ans) : les enfants éblouis par la lumière qui sortait du tronc.

Reformulation avec changement de sens

Un seul cas est attesté chez une fillette francophone qui a transformé la métaphore : au lieu de comparer la lumière à un élément liquide comme dans le texte source, elle donne à l'élément liquide une propriété attachée à la lumière en inversant en quelque sorte le comparant et le comparé. Nous considérons que la reformulation d'une métaphore en une autre métaphore est une procédure de reformulation difficile à réaliser même si l'enfant a fortement simplifié la syntaxe (avec deux informations provenant de la principale : voient, déduite de furent éblouis, éblouissante dérivée de éblouis, et une information provenant de la relative : mer, déduite de inondait) :

Océanie (10 ans) : ils voient une mer éblouissante.

Reformulation par paraphrase formelle (restructuration de la relative)

Cette procédure difficile à produire n'est attestée que chez un enfant francophone, qui, de plus, a reproduit la métaphore :

Léo (10 ans) : à l'intérieur c'est inondé de lumière. 
Tableau 3 : Répartition des types de reformulation de la relative dans la séquence 11

\begin{tabular}{|c|c|c|c|c|c|c|c|}
\hline & \multirow{2}{*}{$\begin{array}{l}4 \text { ans } \\
\text { Fr. } \\
\mathrm{N}=12\end{array}$} & \multicolumn{2}{|c|}{6 ans } & \multicolumn{2}{|c|}{8 ans } & \multicolumn{2}{|c|}{10 ans } \\
\hline & & $\begin{array}{c}\mathrm{Fr} . \\
\mathrm{N}=13\end{array}$ & $\begin{array}{c}\text { All. } \\
\mathrm{N}=10\end{array}$ & $\begin{array}{c}\mathrm{Fr} . \\
\mathrm{N}=15\end{array}$ & $\begin{array}{c}\text { All. } \\
\mathrm{N}=12\end{array}$ & $\begin{array}{c}\text { Fr. } \\
\mathrm{N}=15\end{array}$ & $\begin{array}{c}\text { All. } \\
N=13\end{array}$ \\
\hline Répétitions lacunaires & & & & & $3 / 5$ & & \\
\hline Répétitions complètes & & & & & & & $1 / 4$ \\
\hline Paraphrases sémantiques & & & & & & & $1 / 4$ \\
\hline Paraphrases logiques & & & $1 / 1$ & & $2 / 5$ & $1 / 3$ & $2 / 4$ \\
\hline $\begin{array}{l}\text { Reformulations avec } \\
\text { changement de sens }\end{array}$ & & & & & & $1 / 3$ & \\
\hline $\begin{array}{l}\text { Restructurations de la } \\
\text { relative }\end{array}$ & & & & & & $1 / 3$ & \\
\hline $\begin{array}{l}\text { TOTAL des } \\
\text { reformulations de la } \\
\text { relative / reformulations } \\
\text { de la séquence }\end{array}$ & & & $1 / 10$ & & $5 / 12$ & $3 / 15$ & $4 / 13$ \\
\hline Occurrence d'une relative & & & & & & $1 / 3$ & $1 / 4$ \\
\hline
\end{tabular}

\section{Conclusion}

La différence quantitative entre l'allemand et le français (10 reformulations en allemand, les trois âges confondus c. 3 en français à 10 ans seulement) s'explique par l'absence de métaphore dans le texte allemand. Les francophones n'ont pas pu répéter le texte source. Un seul enfant de 10 ans a produit une paraphrase formelle (restructuration) et c'est le même enfant qui a repris la métaphore.

D'un point de vue sémantique, il semble que la reformulation de l'intensité lumineuse exprimée par le verbe de la principale dans les deux langues et par le verbe métaphorique de la relative en français ait réservé des difficultés aux enfants de 4 (fr.) et 6 ans (fr. et germ.). 
En effet, ce n'est qu'à 8 ans, dans les deux langues, qu'on trouve une trace de cette grande quantité (voir ci-dessus les paraphrases logiques). On peut expliquer ce phénomène par le fait que ce n'est manifestement qu'à 8 ans que les enfants sont capables d'analyser et de décomposer le sens contextuel des verbes : être éblouis/geblendet werden; inonder. Ils peuvent donc " extraire " l'idée d'une lumière très intense. Ce processus de déconstruction-reconstruction du sens correspond à l'hypothèse des matrices analytiques définitoires (MAD) qu'Ibrahim développe depuis 2001, hypothèse qui permet de rendre compte de toutes les reformulations analytiques définitoires complètes et lacunaires que les enfants utilisent relativement à un énoncé source $^{22}$. La MAD de "les enfants furent éblouis par la lumière " serait : les enfants furent l'objet d'un éblouissement produit par une lumière tellement forte qu'ils ne pouvaient plus rien voir $^{23}$.

Le faible poids narratif explique aussi que peu d'enfants aient cherché à reformuler le contenu sémantique de la relative.

Le réemploi d'une relative par un seul enfant germanophone de 10 ans et un seul enfant francophone de même âge va dans le sens d'une plus grande difficulté de traitement de la relative déterminative par rapport à la relative explicative.

Il apparaît que la concentration de phénomènes complexes dans la principale et la relative a réservé de grandes difficultés aux enfants dans les deux langues. Les reformulations par paraphrases logiques des germanophones sont d'autant plus remarquables qu'elles correspondent, d'une part, à des procédures complexes; d'autre part, qu'elles montrent la créativité des enfants. C'est aussi le cas chez Océanie (10 ans) qui a produit une nouvelle métaphore. Du point de vue de la créativité formelle, la restructuration faite par Léo (10 ans) est étonnante.

\footnotetext{
22 Voir aussi Amr H. Ibrahim et Claire Martinot, "Les reformulations matricielles lacunaires des enfants ", Travaux de linguistique, n 48, 2004, p. 21-40. Sonia Gerolimich et coll., "Reformulations définitoires spontanées chez des francophones et des italophones de 6, 8 et 10 ans ", Autour de la définition, 6, éd. Publif@rum, 2009. http://www.publifarum.farum.it/ezine_printarticle.php?art_id=148 http://www.publifarum.farum.it/ezine_printarticle. php?art_id=149.
} 


\section{Reformulations de la relative de la séquence $\mathbf{1 2}$}

[Fr.] Tom et Julie se trouvaient dans un jardin merveilleux où les fleurs semblaient se parler en chantant.

[All.] Tom und Julia befanden sich in einem Wundergarten, wo es schien, als ob die Blumen sangen, wenn sie miteinander redeten.

Remarques générales

La relative explicative de cette séquence comporte deux zones de complexité qui seront analysées successivement. D'une part, la relation hiérarchique entre les deux prédicats : parler, qui est le prédicat principal, et en chantant, qui est le prédicat second, d'autre part, le sémantisme du verbe opérateur épistémique (semblaient/schien).

En français, la construction gérondive est intégrée dans la proposition relative, ce qui correspond à un degré de complexité élevée ${ }^{24}$; en allemand, le degré d'intégration est moindre puisque l'on a trois propositions subordonnées successives ${ }^{25}$. On peut s'attendre à ce que la difficulté d'interprétation (ou de production) soit plus grande en français qu'en allemand. Mais dans tous les cas, les enfants doivent comprendre qu'il n'y a qu'un seul procès : se parler, qui se réalise d'une certaine façon : en chantant.

Le verbe opérateur sembler/scheinen correspond à une modalité épistémique dans laquelle l'énonciateur exprime une illusion perceptive sans se prononcer sur la véracité de la relation prédicative transmise par les fleurs se parlaient en chantant.

Lapport informationnel de la relative dans l'histoire est faible. Le fait que les fleurs se parlent en chantant peut expliquer pourquoi le jardin est merveilleux, mais cela n'a aucune incidence sur les deux protagonistes, ni sur la suite de l'histoire.

\footnotetext{
24 Talmy Givón, Functionalism and Grammar, Amsterdam/Philadelphia, John Benjamins, 1995.

25 De façon à pouvoir comparer l'allemand avec le français du point de vue des informations reformulées, nous désignons par relative en allemand l'énoncé qui commence avec le pronom relatif locatif wo et se termine avec le verbe redeten qui clôt la circonstancielle.
} 


\section{Procédures de reformulation}

Dans les deux langues, le contenu informationnel de la relative est difficile à paraphraser du fait qu'il n'y a qu'un procès bien qu'il y ait deux verbes prédicatifs $\left(\mathrm{V}_{\mathrm{i}}\right.$ : parler/reden $-\mathrm{V}_{\mathrm{j}}$ : chanter/ singen), mais aussi du fait qu'il existe peu de synonymes pour parler et encore moins pour chanter. Jusqu'à 8 ans, dans les deux langues, les enfants ont changé le sens de la prédication complexe les fleurs se parlaient en chantant/die Blumen redeten miteinander, wenn sie sangen [les fleurs parlaient les unes avec les autres quand elles chantaient]. À 10 ans, en revanche, les répétitions (approximatives) de cette prédication sont plus nombreuses que les changements de sens.

Concernant l'expression de la modalité épistémique, elle est absente en français chez les 4 ans et rare chez les 6 ans. En revanche, elle est fréquemment reformulée dès 6 ans chez les germanophones. Les francophones ont produit majoritairement un synonyme lexical (on dirait que, ils ont limpression que, paraissaient, on sentait que, on croyait que), tandis que les germanophones ont produit majoritairement un équivalent grammatical (würden : auxiliaire du subjonctif II, exprimant l'irréel).

Reformulations de la prédication complexe $\left(\mathrm{V}_{\mathrm{i}}-\mathrm{V}_{\mathrm{j}}\right)$ avec changement de sens

Nous considérons qu'il y a un changement de sens lorsque l'un des deux verbes, parler/reden ou chanter/singen est effacé (Gabriel) ou bien lorsque les deux verbes expriment chacun un procès différent (Alexandre), ou encore lorsque les procès sont inversés (Natalie) :

Gabriel (4 ans) : *les fleurs se tendaient se parler/parlaient.

Alexandre (4 ans) : il y avait des fleurs qui se parlaient et qui chantaient.

Alexia (6 ans) : on dirait que les fleurs elles chantaient.

Chloé (8 ans) : c'est comme si les fleurs ils parlaient ensemble.

Marion (10 ans) : il lui semble que les fleurs se parlent entre elles.

Vanessa (6 ans) : es hat so ausgesehen, als würden die Blumen singen [il a semblé que les fleurs chantaient]. 
Tamara (8 ans) : dann haben die Blumen geredet [après les fleurs ont parlé].

Natalie (10 ans) : da haben die Vögel gesungen, als täten sie miteinander sprechen [alors les oiseaux ont chanté comme s'ils parlaient ensemble] ${ }^{26}$.

Reformulations par répétition approximative de la prédication complexe

Les variations portent en français sur le temps et le mode de se parler (qui n'est jamais à l'infinitif sauf chez Romain), ainsi que sur la présence ou non du pronom réfléchi se devant parler. En allemand, elles portent sur le temps et sur l'ordre des subordonnées :

Achille ( 6 ans) : les fleurs parlaient en chantant.

Chloé (6 ans) : où ils ont l'impression que les fleurs elles se parlent en chantant.

Solène (8 ans) : on disait même qu'elles parlaient en chantant.

Barbara (10 ans) : où on avait l'impression que les fleurs se parlaient en chantant.

Romain (10 ans) : il voit des fleurs parler en chantant.

Sebastian (10 ans) : da schien es so, *als ob die Blumen singen, wenn sie miteinander reden [alors il semblait que c'était comme si les fleurs chantaient quand elles parlaient les unes avec les autres - les deux derniers verbes auraient dû être au prétérit].

Reformulations par paraphrase sémantique

Ces paraphrases résultent de l'emploi d'un synonyme du verbe source reden/parler, repris par sprechen, et du verbe source parler repris par discuter:

Mona (6 ans) : die Blumen, wenn die sprechen, würden die so singen [les fleurs quand elles parlent, c'est comme si (würden: auxiliaire de l'irréel) elles chantent].

Simone (8 ans) : wo es sich anhört, dass die Blumen singen, wenn sie sprechen [où on entend, que les fleurs chantent quand elles parlent].

26 Dans l'évaluation du changement de sens, nous n'avons pas tenu compte de la confusion entre Vögel/oiseaux et Blumen/fleurs mais de l'inversion des procès. 
Alexandre (8 ans) : où on sentait qu'il y avait des fleurs qui discutaient en chantant.

\section{Reformulations par paraphrase formelle}

Il n'y a qu'une attestation, chez un enfant de 4 ans, de ce type complexe de paraphrase. Margot introduit dans la relative en où une fausse relative en il y a ...qui, et permute les deux verbes prédicatifs ${ }^{27}$ : Margot (4 ans) : où il y avait des fleurs qui chantaient en parlant.

Expression de la modalité « semblaient/es schien [il semblait]»

L'expression de cette modalité est difficile pour les enfants francophones jusqu'à l'âge de 8 ans. À 4 ans, aucun enfant n'a tenté de reformuler la modalité, à 8 ans les tentatives sont nombreuses, mais trois enfants ont encore modifié le sens du verbe. À 10 ans, en revanche, les quatre enfants qui ont reformulé semblaient ont produit un synonyme ou une restructuration (voir Marion) :

Chloé (8 ans) : c'est comme si les fleurs ils parlaient ensemble.

Marion (10 ans) : il lui semble que les fleurs se parlent entre elles.

Léo (10 ans) : où il avait l'impression que les fleurs se parlaient en chantant.

Chez les enfants germanophones, la modalité es schien a été soit effacée (peu de cas) soit reformulée sans changement de sens dès 6 ans, avec l'auxiliaire de l'irréel würden ettou la conjonction als (ob) [comme si]. À 6 et 8 ans, l'impression exprimée par cette modalité épistémique est donc grammaticalisée et est souvent accompagnée d'un verbe de perception : sich anhören [entendre]. Ce n'est qu'à 10 ans, cependant, que es schien est répété (deux cas) et qu'un autre verbe épistémique glauben [croire] est utilisé :

$27 \quad$ La relation sémantique qui relie parler et chanter fait que l'ordre des verbes est indépendant du sens de l'ensemble $:$ parler en chantant $=$ chanter en parlant. On a la même situation quand un des deux verbes décrit comment se déroule le procès énoncé par l'autre verbe : il marche en boitant = il boite en marchant, il nage en crawlant = il crawle en nageant, il skie en godillant = il godille en skiant... Voir Claire Martinot, "Dans quels cas les constructions $V$ en $V_{-a n t}$ sont-elles ambiguës? ", Bibliothèque de Faits de langue (sous presse $\mathrm{b}$ : Berne, Peter Lang, Bibliothèque de Faits de langue). 
Kimberly (6 ans) : das hörte sich so an, *als die Blume rede [on entendait comme si les fleurs parlaient - Blume aurait dû avoir la forme pluriel : Blumen et rede aurait dû avoir la forme redet (3S) ou reden (3P), la forme conjuguée du verbe aurait dû suivre als].

Florian (10 ans) : es schien, dass sie *inge, wenn sie miteinander reden [il semblait qu'elles chantaient quand elles parlaient ensemble - singe aurait dû avoir la forme pluriel singen, les formes verbales singen et reden auraient dû être au même temps, prétérit, que le verbe principal].

Lukas (10 ans) : *als sie hingehe, glaubte man, dass die Blumen sangen, wenn sie sprach [quand ils y vont, on croyait, que les fleurs chantaient - hingehe auraît dû être au pluriel et au prétérit, la différence de sujet entre sie et man révèle une difficulté dans la cohérence].

Occurrence d'une relative, d'un gérondif (fr.) ou de plusieurs subordonnées (all.)

Les occurrences de relatives en allemand restent marginales. En français, bien que peu nombreuses, elles augmentent en fonction de l'âge. L'emploi d'un gérondif en français est attesté dans les mêmes proportions : une occurrence à 4 ans, deux à 6 ans, trois à 8 et 10 ans (tableau 4). En allemand, la plupart des enfants ont employé au moins une subordonnée, soit introduite par wenn [quand], soit par als (ob) [comme si] et quelques-uns ont introduit une complétive à la suite d'un verbe de perception (EvaMaria, ci-dessous). Les suites hypotaxiques semblent être maîtrisées par les enfants germanophones de 6 ans :

Eva-Maria (6 ans) : *haben sie gesehen, dass die Blumen \# mit sich reden und singen [ils ont vu que les fleurs se parlent (avec elles-mêmes) et chantent - les deux derniers verbes auraient dû être au prétérit].

Nadine (6 ans) : *die Blumen redeten miteinander, dass es so klingt, als singen sie [les fleurs parlaient ensemble si bien que ça sonnait comme si elles chantaient - comme précédemment, les deux derniers verbes auraient dû être au prétérit]. 
Tableau 4 : Répartition des types de reformulation de la relative dans la séquence 12

\begin{tabular}{|c|c|c|c|c|c|c|c|}
\hline & \multirow{2}{*}{\begin{tabular}{|c}
4 ans \\
Fr. \\
$\mathrm{N}=12$
\end{tabular}} & \multicolumn{2}{|c|}{6 ans } & \multicolumn{2}{|c|}{8 ans } & \multicolumn{2}{|c|}{10 ans } \\
\hline & & $\begin{array}{c}\mathrm{Fr} . \\
\mathrm{N}=14\end{array}$ & $\begin{array}{c}\text { All. } \\
\mathrm{N}=12\end{array}$ & $\begin{array}{c}\mathrm{Fr} . \\
\mathrm{N}=15\end{array}$ & $\begin{array}{c}\text { All. } \\
\mathrm{N}=13\end{array}$ & $\begin{array}{c}\text { Fr. } \\
N=14\end{array}$ & $\begin{array}{c}\text { All. } \\
\mathrm{N}=15\end{array}$ \\
\hline $\begin{array}{l}\text { Changements de } \\
\text { sens de la } \\
\text { prédication } \\
\text { complexe }\left(\mathrm{V}_{\mathrm{i}}-\mathrm{V}_{\mathrm{j}}\right)\end{array}$ & $3 / 4$ & $2 / 4$ & $5 / 7$ & $5 / 8$ & $1 / 2$ & $2 / 5$ & $2 / 6$ \\
\hline $\begin{array}{l}\text { Répétitions } \\
\text { approximatives de } \\
\left(\mathrm{V}_{\mathrm{i}}-\mathrm{V}_{\mathrm{j}}\right)\end{array}$ & & $2 / 4$ & $1 / 7$ & $2 / 8$ & & $3 / 5$ & $3 / 6$ \\
\hline $\begin{array}{l}\text { Paraphrases } \\
\text { sémantiques de } \\
\left(V_{i}-V_{j}\right)\end{array}$ & & & $1 / 7$ & $1 / 8$ & $1 / 2$ & & $1 / 6$ \\
\hline $\begin{array}{l}\text { Paraphrases } \\
\text { logiques }\end{array}$ & $1 / 4$ & & & & & & \\
\hline $\begin{array}{l}\text { TOTAL des } \\
\text { reformulations de } \\
\left(\mathrm{V}_{\mathrm{i}}-\mathrm{V}_{\mathrm{i}}\right) / \\
\text { reformulations de la } \\
\text { séquence }\end{array}$ & $4 / 12$ & $4 / 14$ & $7 / 12$ & $8 / 15$ & $2 / 13$ & $5 / 14$ & $6 / 15$ \\
\hline $\begin{array}{l}\text { Changements de } \\
\text { sens de la modalité } \\
\left(\mathrm{V}_{\mathrm{op}}\right)\end{array}$ & $4 / 4$ & $2 / 4$ & $2 / 7$ & $3 / 8$ & $1 / 2$ & $1 / 5$ & $1 / 6$ \\
\hline $\begin{array}{l}\text { Synonymes de } \mathrm{V}_{\mathrm{op}} \text {, } \\
\text { restructurations, } \\
\text { répétitions }\end{array}$ & & $2 / 4$ & $5 / 7$ & $4 / 8$ & $1 / 2$ & $4 / 5$ & $5 / 6$ \\
\hline $\begin{array}{l}\text { Occurrences d'une } \\
\text { relative - Nombre } \\
\text { de subordonnées } \\
\text { (all) }\end{array}$ & $1 / 4$ & $2 / 4$ & $\begin{array}{r}0 / 7 \\
2 \times 2 \text { sub }\end{array}$ & $3 / 8$ & $\begin{array}{l}1 / 2 \text { suivie } \\
\text { de } 2 \text { sub. }\end{array}$ & $3 / 5$ & $\begin{array}{r}0 / 6 \\
4 \times 2 \text { sub } \\
1 \times 1 \text { sub }\end{array}$ \\
\hline
\end{tabular}




\section{Conclusion}

Les changements de sens concernant la reformulation de se parler en chantant restent présents même chez les enfants de 10 ans, ce qui est inhabituel par rapport aux séquences précédentes. Ce résultat vient $\mathrm{du}$ fait que les enfants dissocient parler/reden et chanter/singen. La difficulté vient probablement de la relation sémantique entre les deux verbes qui n'est pas comprise ou est trop difficile à reformuler : l'un des deux verbes décrit le déroulement de l'autre. Cette relation, fortement grammaticalisée en français, présente une ambiguïté, par conséquent une difficulté d'interprétation ${ }^{28}$. En allemand, il n'y a a priori pas d'ambiguïté d'autant que l'illusion que les fleurs chantent est marquée deux fois : par l'opérateur de modalité es schien/il semblait qui ne porte que sur la proposition suivante et par la conjonction als ob/comme si qui concerne uniquement le verbe sangen/ chantaient: es schien, als ob die Blumen sangen lil semblait comme si les fleurs chantaient. Pourtant les changements de sens sont en moyenne aussi nombreux en allemand qu'en français, et la moyenne de reformulation de la relative de la séquence 12 est la même dans les deux langues (tableau 6). Dans cette séquence, c’est avant tout la difficulté sémantique qui rend la reformulation de la relative difficile. Cette difficulté est double en français : relation entre parler et chanter, sens de sembler.

L'expression de la modalité épistémique (grammaticalisée en allemand) semble être maîtrisée dès 6 ans chez les germanophones, alors qu'elle ne l'est qu'à 10 ans chez les francophones. Le français ne dispose pas d'un auxiliaire équivalent à würden; les jeunes locuteurs francophones doivent donc, dans cette tâche de restitution, soit répéter sembler, soit le reprendre par un synonyme lexical.

La quasi-absence de relatives dans les reformulations des enfants germanophones s'explique, comme pour la séquence 6 ,

$\overline{28}$ La construction gérondive est ambiguë dans la mesure où ce n'est que la relation sémantique entre le verbe conjugué et le verbe gérondivé qui indique s'il s'agit d'un seul procès ou de deux procès simultanés : par exemple, il parle en dormant $=2$ procès $\mathrm{c}$. il marche en courant $=1$ procès (Claire Martinot, sous presse b : Berne, Peter Lang, Bibliothèque de Faits de langue, op. cit). 
par la possibilité d'employer un adverbe locatif anaphorique là où le français utilise les pronoms relatifs : sur lequel (séquence 6) et où (séquence 12). De plus, l'adverbe est plus facile à utiliser puisqu'il est moins contraint par le cotexte que les pronoms relatifs.

\section{Reformulations de la relative de la séquence 13}

[Fr.] Tom a répondu : « je veux apprendre à parler avec les oiseaux qui savent tout ce qui se passe dans le ciel, avec les poissons qui savent tout ce qui se passe dans l'eau et avec les fourmis qui savent tout ce qui se passe sur la terre ».

[All.] Tom antwortete : " ich möchte lernen mit den Vögeln zu reden, die all das wissen, was im Himmel passiert, mit den Fischen, die all das wissen, was im Wasser passiert, mit den Ameisen, die all das wissen, was auf der Erde passiert".

Remarques générales

Les trois relatives explicatives de cette séquence sont constituées d'un verbe prédicatif savent / wissen suivi d'une complétive enchâssée. D'un point de vue syntaxique, on peut considérer que l'enchâssement d'une complétive dans une relative est un phénomène complexe ${ }^{29}$, on verra si ce phénomène est difficile à reformuler. D'un point de vue sémantique, le fait que le sujet de la relative (oiseaux/Vögel, poissons/Fische, fourmis/Ameisen) soit différent du sujet de la principale (Tom) constitue un autre phénomène complexe : qui fait quoi? (séquence 6). En revanche, la triple reprise de l'enchaînement avec les $N$ qui/mit den $N(. .$.$) ,$ die a souvent permis aux enfants de corriger la deuxième ou la troisième reprise, et en tous cas a favorisé la mémorisation de cette séquence (tableau 6). Cette triple reprise est un facteur facilitateur qui peut contrebalancer la complexité syntaxique et sémantique.

29 Talmy Givón, op. cit. 


\section{Procédures de reformulation}

Dans les deux langues, on peut faire le constat que jusqu'à 8 ans, la reformulation de cette séquence a posé de nombreux problèmes qui se manifestent par des changements de sens et des répétitions lacunaires d'une partie de la relative (dont la complétive) qui conduisent à des énoncés agrammaticaux. En revanche, à 10 ans, la moitié des enfants francophones produit une paraphrase, tandis que les reformulations des germanophones comportent encore des changements de sens.

Pour la première fois, la relative explicative a été reformulée par certains enfants (8 et 10 ans) par une causative. C'est aussi dans cette séquence que les reformulations de la relative par une relative (ou une causative équivalente) ont été les plus fréquentes, dans les deux langues.

On comptabilise et on analyse uniquement la reformulation de la relative la mieux réussie.

Reformulations de la relative avec changement de sens complet

Camille ( 4 ans) : j'aime bien tout ce qui se passe.

Antoine ( 4 ans) : et puis Tom il lui a dit de lui dire comment les poissons nagent dans l'eau.

Changement de sens provenant de la non-distinction des sujets

Jusqu'à l'âge de 8 ans, dans les deux langues, la plupart des enfants ont considéré que le sujet de la relative était le même que celui de la principale, ce qui entraîne un changement de sens. Chez les francophones, la relative a été reformulée par une infinitive introduite par pour, et chez les germanophones, par une conjonctive équivalente en damitlafin que. Cette interprétation est cohérente avec ce que dit la dernière séquence de l'histoire : Et depuis ce jour, Tom est devenu un enfant extrêmement savant. Il n'empêche que l'information selon laquelle les trois catégories d'animaux savent tout ce qui se passe dans leur univers doit être énoncée, puisque c'est cette information qui justifie que Tom veuille apprendre la langue de ces animaux. À l'inverse, lorsque 
les enfants reformulent en disant pour savoir ce qu'il y a dans l'eau, ou l'équivalent allemand, ils effacent le fait que les animaux savent tout ce qui se passe chez eux. Ce n'est qu'à 10 ans, que les enfants comprennent qu'il s'agit bien d'une transmission par la langue d'un savoir :

Alexandre ( 4 ans) : il a demandé de parler aux fourmis pour voir il y a quoi sur la terre.

Daphné (6 ans) : Tom lui *disa : « je veux apprendre à parler avec les oiseaux pour voir tout ce qui se passe dans le ciel (...)».

Ilan (8 ans) : Tom voulait savoir parler aux oiseaux pour savoir tout ce qui se passe dans le ciel.

Kimberly (6 ans) : er will wissen, was im Himmel geschehen ist [il veut savoir ce qui s'est passé dans le ciel].

Anja (8 ans) : dann hat er sich gewünscht, dass er mit den *Vögel sprechen kann, damit er weiss, was oben in der Luft passiert [après il a souhaité qu'il puisse parler avec les oiseaux afin qu'il sache ce qui se passe là-haut dans l'air - Vögel aurait dû être terminé par la marque du datif pluriel $n]$.

Lukas (10 ans) : Tom wünschte sich, dass er mit den Vögeln sprechen konnte, damit er wusste, was alles im Himmel passiert [Tom souhaitait qu'il puisse parler avec les oiseaux afin qu'il sache tout ce qui se passait dans le ciel].

\section{Reformulations par répétition}

\section{Répétitions partielles lacunaires entraînant une agrammaticalité}

Ce type de reformulation n'est attesté que dans cette séquence, manifestant à la fois la difficulté d'interprétation et la difficulté syntaxique de l'enchaînement. Il est plus souvent attesté chez les enfants germanophones (tableau 5):

Antoine ( 4 ans) : je veux parler aux oiseaux * tout ce qui se passe dans l'eau.

Vanessa (6 ans) : ich würde gerne mit den Tieren reden (...) mit den Fischen, *was da passiert [j'aimerais bien parler avec les animaux (...) avec les poissons ce qui se passe là]. 
Julian (6 ans) : da hat sich der eine, der Tom, gewünscht, dass er mit den Vögeln reden konnte, *im Wasser was passiert [alors l'un, Tom, a souhaité qu'il puisse parler avec les oiseaux, il se passe quelque chose dans l'eau].

Simone (8 ans) : dass ich mit den Vögeln sprechen kann, * was im Himmel passiert [que je puisse parler avec les oiseaux ce qui se passe dans le ciel].

Anna-Maria (10 ans) : er möchte mit den Vögeln sprechen können, *all das was im Himmel passiert [il voudrait pouvoir parler avec les oiseaux tout ce qui se passe dans le ciel].

\section{Répétitions complètes}

La relative ne se prête pas à des reformulations paraphrastiques très variées, ce qui explique la proportion relativement élevée de répétitions chez les enfants de 10 ans, qui, de façon générale, ne privilégient plus la répétition :

Marc (10 ans) : je veux savoir parler (...) aux fourmis qui savent tout ce qui se passe sur la terre.

Hanna (10 ans): ich möchte die Vögel verstehen, die alles wissen, was im Himmel passiert [je voudrais comprendre les oiseaux qui savent tout ce qui se passe dans le ciel].

Reformulations par paraphrase sémantique

Quelques paraphrases sémantiques sont attestées chez les enfants francophones seulement qui ont proposé un synonyme de savoir: connaître, de se passe : il y a, et même de ciel : l'air :

Edward (6 ans) : les fourmis qui connaissent la terre.

Gaël (6 ans) : je veux savoir parler aux oiseaux qui savent tout ce qu'il $\mathrm{y}$ a dans le ciel.

Théo (10 ans) : parler aux oiseaux parce qu'ils connaissent tout de l'air.

Reformulations par paraphrase formelle (restructuration)

La quasi-totalité des restructurations correspond à une réduction de la complétive en un complément prépositionnel (dans l'air) über den Himmel), ce que nous considérons comme une simplification syntaxique : 
Océane (8 ans) : Tom demande de savoir parler aux oiseaux parce qu’ils savent tout dans l'air.

Max (10 ans) : ich möchte mit den *Vögel sprechen, die alles wissen über den Himmel [je voudrais parler avec les oiseaux qui savent tout sur le ciel - Vögel aurait dû prendre la marque du datif pluriel $n]$.

Un cas de restructuration maintenant le même degré de complexité linguistique est attesté chez un enfant de 10 ans qui a restructuré le verbe de la principale : Parler avec les $N$ qui en parler à $N$ de $P$, ce qui transforme le verbe de la relative savent en complément du verbe principal :

Maxime (10 ans) : je voudrais pouvoir parler aux poissons de tout ce qu'ils savent dans l'eau

Reformulation par paraphrase logique

Quelques paraphrases logiques sont attestées chez les enfants germanophones seulement. Philipp a modifié le point de vue en effaçant le début de la séquence 13 pour dire que les animaux racontent ce qui se passe dans leur univers, ce qui présuppose qu'ils le savent :

Philipp (6 ans) : und dann erzählten die Ameisen, was auf der Erde passiert [et après les fourmis racontaient ce qui se passe sur la terre].

Katrin et Lukas ont explicité le fait que les oiseaux disent ou racontent quelque chose :

Katrin (8 ans): und dann hat er sich gewünscht, dass er mit den Vögeln sprechen kann, dass die sagen können, was am Himmel passiert [et après il a souhaité qu'il puisse parler aux oiseaux afin qu'ils puissent dire ce qui se passe dans le ciel].

Lukas (8 ans) : dann hat er sich gewünscht, dass er mit den Vögeln reden kann, dass die ihm dann erzählen können, was oben am Himmel passiert [après il a souhaité qu'il puisse parler avec les oiseaux afin qu'ils puissent après lui raconter ce qui se passe dans le ciel]. 
Tableau 5 : Répartition des types de reformulation de la relative dans la séquence 13

\begin{tabular}{|c|c|c|c|c|c|c|c|}
\hline & 4 ans & \multicolumn{2}{|c|}{6 ans } & \multicolumn{2}{|c|}{8 ans } & \multicolumn{2}{|c|}{10 ans } \\
\hline & $\begin{array}{c}\text { Fr. } \\
N=14\end{array}$ & $\begin{array}{c}\mathrm{Fr} . \\
\mathrm{N}=14\end{array}$ & $\begin{array}{c}\text { All. } \\
\mathrm{N}=11\end{array}$ & $\begin{array}{c}\text { Fr. } \\
N=14\end{array}$ & $\begin{array}{c}\text { All. } \\
N=13\end{array}$ & $\begin{array}{c}\mathrm{Fr} . \\
\mathrm{N}=15\end{array}$ & $\begin{array}{c}\text { All. } \\
\mathrm{N}=11\end{array}$ \\
\hline $\begin{array}{l}\text { Changements de } \\
\text { sens de la relative }\end{array}$ & $8 / 10$ & $9 / 14$ & $2 / 8$ & $8 / 12$ & $5 / 12$ & $1 / 13$ & $3 / 10$ \\
\hline $\begin{array}{l}\text { Répétitions lacunaires } \\
=>\text { agrammaticalité }\end{array}$ & $1 / 10$ & & $4 / 8$ & & $2 / 12$ & $1 / 13$ & $1 / 10$ \\
\hline Répétitions complètes & $1 / 10$ & $2 / 14$ & $1 / 8$ & $1 / 12$ & $1 / 12$ & $3 / 13$ & $3 / 10$ \\
\hline $\begin{array}{l}\text { Paraphrases } \\
\text { sémantiques }\end{array}$ & & $3 / 14$ & & $1 / 12$ & & $3 / 13$ & \\
\hline $\begin{array}{l}\text { Restructurations de la } \\
\text { relative }\end{array}$ & & & & $2 / 12$ & $2 / 12$ & $5 / 13$ & $2 / 10$ \\
\hline Paraphrases logiques & & & $1 / 8$ & & $2 / 12$ & & $1 / 10$ \\
\hline $\begin{array}{l}\text { TOTAL des } \\
\text { reformulations de la } \\
\text { relative/reformulations } \\
\text { de la séquence }\end{array}$ & $10 / 14$ & $14 / 14$ & $8 / 11$ & $12 / 14$ & $12 / 13$ & $13 / 15$ & $10 / 11$ \\
\hline $\begin{array}{l}\text { Occurrences d'une } \\
\text { relative }\end{array}$ & $1 / 10$ & $4 / 14$ & $1 / 8$ & $3 / 12$ & $2 / 12$ & $7 / 13$ & $3 / 10$ \\
\hline $\begin{array}{l}\text { Occurrences d'une } \\
\text { causative }\end{array}$ & & & & $1 / 12$ & $3 / 12$ & $2 / 13$ & $2 / 10$ \\
\hline
\end{tabular}

\section{Conclusion}

Cette séquence, aussi souvent reformulée que la séquence 6, malgré une plus grande complexité syntaxique, partage avec celle-ci une pertinence narrative importante. La complexité syntaxique et sémantique de la séquence 13 explique que les locuteurs ont majoritairement modifié le sens jusqu'à 8 ans dans les deux langues, ce qui n'est pas le cas pour la séquence 6 . 
Comme pour la séquence 12 , les répétitions sont plus nombreuses à 10 ans que chez les plus jeunes, du fait que les verbes prédicatifs des deux séquences sont difficilement paraphrasables (parler/reden, chanter/singen, savoir/wissen).

L'augmentation des équivalences sémantiques (paraphrases sémantiques, formelles et logiques) est très nette chez les francophones entre 8 et 10 ans; elle l'est moins chez les germanophones.

Cette séquence est la seule dans laquelle la relative source est reformulée par une causative, dès 8 ans dans les deux langues.

Le réemploi d'un pronom relatif en allemand augmente légèrement entre 6 et 10 ans. En français, l'augmentation est significative entre 8 et 10 ans. On remarquera que si le taux de réemploi d'une relative est le même chez les francophones de 10 ans pour la séquence 6 et la séquence 13, l’augmentation n'a lieu qu'à 10 ans pour la séquence 13 et non à 8 ans, comme c'est le cas pour la séquence 6 . Ce résultat corrobore la difficulté qu'ont rencontrée les enfants lors de la reformulation de la séquence 13, malgré l'effet facilitateur de la construction avec les $N$, qui savent tout ce qui... trois fois reprise.

Il semblerait que l'augmentation des relatives ou des causatives soit corrélée à l'augmentation des équivalences sémantiques, ce qui plaide en faveur d'une analyse combinée du sens et de la syntaxe, et non pas du seul niveau de constatation que les pronoms relatifs sont en augmentation en fonction de l'âge.

Conclusion générale

La question de savoir s'il y a une corrélation systématique entre un phénomène décrit par les linguistes comme complexe et la difficulté rencontrée par les jeunes locuteurs à produire ce phénomène dans le contexte d'une restitution d'histoire se révèle redoutable. En effet, comme nous l'avons déjà signalé, les difficultés éventuelles des jeunes locuteurs à reformuler les relatives du texte source ne se limitent pas à la construction d'une phrase introduite par un pronom relatif. De nombreux autres phénomènes syntaxiques et sémantiques complexifient les énoncés contenant une relative. Plus ces phénomènes sont nombreux et 
concentrés, plus la reformulation se révèle difficile, mais la nature de ces phénomènes doit aussi être prise en compte. À ces phénomènes s'ajoute la pertinence de l'information exprimée dans la relative par rapport au déroulement de l'histoire. Une pertinence faible rend la reformulation plus difficile. À l'inverse, la redondance d'une construction donnée comme complexe peut faciliter la tâche de restitution.

Nous avons tenté de hiérarchiser les difficultés des locuteurs en fonction de critères quantitatifs (nombre de reformulations de la relative, par exemple, voir tableau 6) et qualitatifs (les reformulations paraphrastiques sont plus difficiles à produire que les répétitions), mais il semble pour le moins hasardeux de hiérarchiser les phénomènes linguistiques, qu'empiriquement les locuteurs adultes s'accordent à considérer comme complexes.

L'analyse des procédures de reformulation pourrait bien se révéler être un outil d'évaluation fiable du rapport entre difficulté du locuteur et complexité linguistique dans la mesure où ce type d'analyse examine toutes les transformations produites par un locuteur entre un énoncé source et un énoncé reformulé.

Le tableau 6 montre que les taux de reformulation sont très différents d'une relative à l'autre, mais fort comparables entre les deux langues.

Les relatives sources les plus reformulées sont de niveau de complexité très différent (séquences 6 et 13), en revanche, elles apportent toutes deux une information narrative importante. Les relatives les moins reformulées sont les deux relatives déterminatives (séquences 4 et 11). Mais la concentration, en français, de différents phénomènes complexes dans la relative de la séquence 11 ne permet pas de distinguer le rôle de chaque phénomène dans la difficulté rencontrée par les enfants. En allemand, toutefois, le fait que cette relative soit beaucoup moins reformulée que les quatre relatives explicatives (séquences 1, 6, 12 et 13) peut être imputable au statut sémantique de cette relative. 
Tableau 6 : Nombre et taux de reformulations, toutes procédures confondues, des relatives du texte source par rapport au nombre d'enfants ayant restitué tout ou partie de la séquence

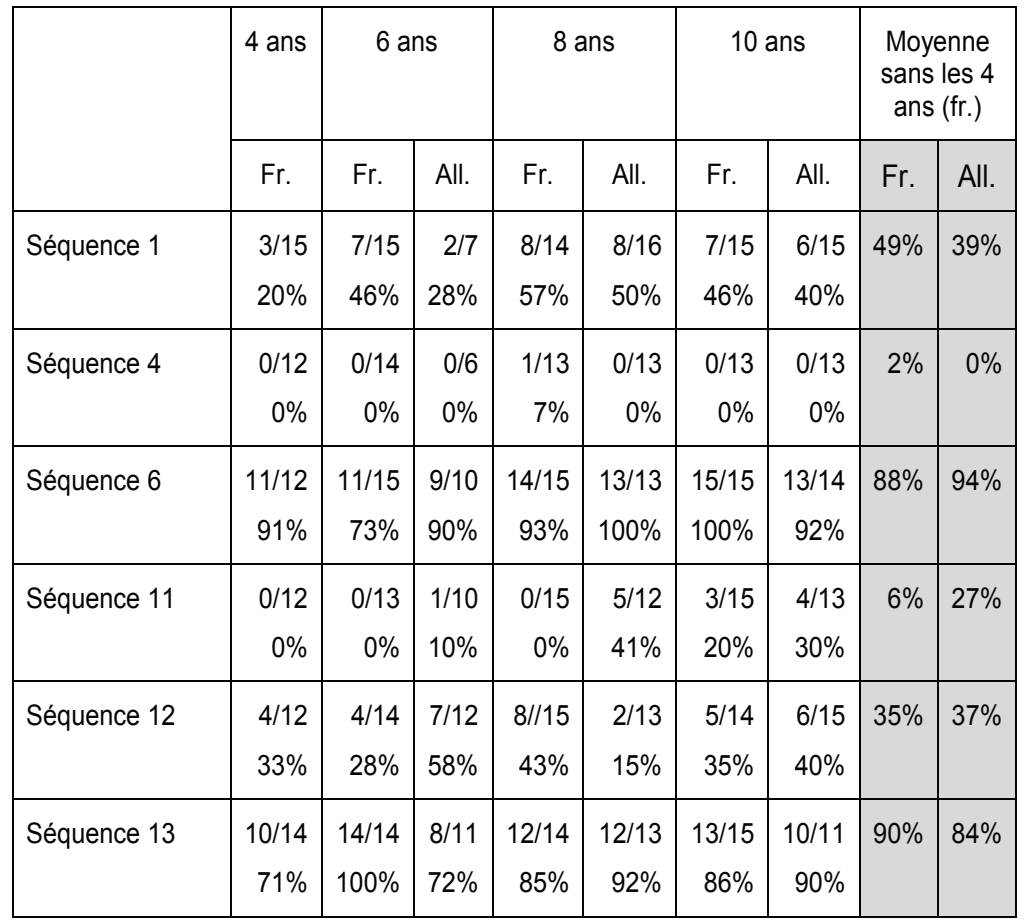

La reformulation des relatives sources par des relatives concerne avant tout les séquences 6 et 13 et, dans une moindre mesure, les séquences 1 et 12 chez les francophones. Mais les relatives reformulées par des relatives ou des causatives apparaissent tout de même plus d'une fois par tranche d'âge dans la séquence 13, chez les enfants germanophones.

Le faible taux de réutilisation d'un pronom relatif par les enfants germanophones relativement aux enfants francophones n'implique pas que les premiers maîtrisent moins bien le système des relatives, mais que les relatives sont beaucoup moins utilisées en allemand. 
Nous espérons avoir montré que l'analyse du différentiel attesté entre l'énoncé source et l'énoncé reformulé est un mode d'analyse transférable d'une langue à l'autre. Les modes de reformulation complexes comme les paraphrases logiques, les restructurations et, dans certains cas, les paraphrases sémantiques sont attestés dans les deux langues à partir de 8 ans. Cet âge semble marquer le passage d'un stade acquisitionnel à un autre, dans la gestion de la complexité linguistique, mais aussi dans la gestion des difficultés réservées par la tâche de reformulation d'une histoire.

\section{Bibliographie}

Dahl, Östen, The Growth and Maintenance of Linguistic Complexity, Amsterdam / Philadelphia, Benjamins, 2004.

Dasinger, Lisa et Cecile Toupin, «The Development of Relative Clause Function in Narrative ", dans Ruth A. Berman et Dan I. Slobin (dir.), Relating Events in Narrative. A Crosslinguistic Developmental Study, Hillsdale, New Jersey, Lawrence Erlbaum, 1994, p. 457-515.

Diessel, Holger, The acquisition of complex sentences, Cambridge, Cambridge University Press, 2004.

Gerolimich, Sonia, Claire Martinot et Michele De Gioia, « Reformulations définitoires spontanées chez des francophones et des italophones de 6, 8 et 10 ans ", Autour de la définition, 6, éd. Publif@rum, 2009. http://www.publifarum.farum.it/ezine_printarticle.php?art_id=148. http://www.publifarum.farum.it/ezine_printarticle.php?art_id=149.

Givón, Talmy, Functionalism and Grammar, AmsterdamPhiladelphia, John Benjamins, 1995.

Harris, Zellig S., Language and Information, New York, Columbia University Press, 1988.

Heylighen, Francis, "Five questions on complexity ", dans Carlos Gershenson (dir.), Complexity: 5 questions, Copenhagu, Automatic Press/VIP, 2008, en ligne: http://arxiv.org/ftp/nlin/papers/0702/0702016. pdf (consulté en août 2012). 
Ibrahim, Amr H., "Les conditions de la prédication dans les langues ", dans Amr H. Ibrahim (dir.), Prédicats, prédication et structures prédicatives, Paris, Cellule de Recherche en Linguistique, 2009, p. 12-49.

Ibrahim, Amr H. et Claire Martinot, "Les reformulations matricielles lacunaires des enfants ", Travaux de linguistique, $\mathrm{n}^{\circ}$ 48, 2004, p. 21-40.

Kleiber, Georges, «Relatives restrictives / relatives appositives : dépassement $(s)$ autorisé(s) ", Langages, n 88, 1987, p. 41-43.

Martinot, Claire, La reformulation dans des productions orales de définitions et explications. (Enfants de maternelle), Thèse de Doctorat, Université Paris VIII, 1994.

Martinot, Claire, "Étude comparative des processus de reformulation chez des enfants de 5 à 11 ans ", Langages, $n^{\circ}$ 140, 2000, p. 92-123.

Martinot, Claire, "Reformulations paraphrastiques et stades d'acquisition en français langue maternelle ", Cahiers de praxématique, n 52, 2009, p. 29-57.

Martinot, Claire, "Reformulation et acquisition de la complexité linguistique ", Travaux de linguistique, n 61, 2010, p. 63-96.

Martinot, Claire, "La reformulation : de la construction du sens à la construction des apprentissages ", (sous presse a $:$ http://www.revuecorela.org ).

Martinot, Claire, "Dans quels cas les constructions $V$ en $V_{-a n t}$ sont-elles ambiguës? ", lieu, Bibliothèque de Faits de langue (sous presse b : Berne, Peter Lang, Bibliothèque de Faits de langue).

Martinot, Claire, Jelena Kuvac-Kraljevic, Tomislava Bosnjak-Botica et Lilian Chur, "Prédication principale $v s$ seconde à l'épreuve des faits d'acquisition ", dans Amr H.Ibrahim (dir.), Prédicats, prédication et structures prédicatives, Paris, Cellule de Recherche en Linguistique, 2009, p. 50-81.

Miestamo, Matti, "Grammatical Complexity in a Cross-Linguistic Perspective ", dans Matti Miestamo, Kaius Sinnemäki et Fred Karlsson (dir.), Language Complexity: Typology, Contact, Change, Amsterdam/ Philadelphia, Benjamins, 2008, p. 23-42. 


\section{Annexe 1}

\section{Tom et Julie}

1. Ce matin-là, la maîtresse est arrivée dans la cour de l'école plus tard que d'habitude. Elle tenait par la main une petite fille que personne n'avait encore jamais vue.

2. Arrivée en classe, la maîtresse a dit : "Les enfants, je vous présente votre nouvelle camarade, elle s'appelle Julie. Tom, la place est libre à côté de toi, Julie sera ta voisine, sois bien gentil avec elle! »

3. Tom était fou de joie à l'idée d'avoir peut-être une nouvelle amie. Le soir, chez lui, il a fabriqué une petite boîte ronde, rouge et dorée, pour Julie.

4. Le lendemain matin, dans la cour de l'école, Tom guettait l'arrivée de sa nouvelle petite voisine. Dès qu'il l'a aperçue, il s'est dirigé vers la fillette et lui a tendu la boîte qu'il avait fabriquée pour elle, la veille.

5. Julie aimait tellement cette boîte qu'elle la prenait toujours avec elle. Quand la maîtresse disait : "Sortez vos affaires! », Julie posait délicatement la boîte entre Tom et elle, sur leur table de travail.

6. Un jour, Julie chuchota à Tom : " Ouvre la boîte! » Tom souleva le couvercle et découvrit un morceau de papier sur lequel Julie avait écrit : " Je t'attends ce soir à $8 \mathrm{~h}$, sous le gros arbre, à l'entrée de la forêt ".

7. Tom avait un peu peur parce qu'il lui était interdit d'aller dans la forêt, surtout la nuit.

8. Mais à $8 \mathrm{~h}$ du soir, il était tout de même au rendez-vous, Julie l'attendait déjà.

9. Sans dire un mot, la petite fille prit la main de Tom et frappa trois fois sur le tronc du gros arbre.

10. Au bout de quelques minutes, les enfants entendirent un grincement. L'arbre était en train de tourner sur lui-même.

11. Tout à coup, le tronc s'ouvrit et les enfants furent éblouis par la lumière qui inondait l'intérieur de l'arbre. Ils firent quelques pas et l'arbre se referma derrière eux 
12. Tom et Julie se trouvaient dans un jardin merveilleux où les fleurs semblaient se parler en chantant. Alors Julie dit à Tom : "Viens, traversons le jardin, il y a une grande fête pour toi, ce soir. Jusqu'à minuit, tu as le droit de demander à notre Roi tout ce que tu veux".

13. Tom a répondu : "Je veux apprendre à parler avec les oiseaux qui savent tout ce qui se passe dans le ciel, avec les poissons qui savent tout ce qui se passe dans l'eau et avec les fourmis qui savent tout ce qui se passe sur la terre ".

14. Et depuis ce jour, Tom est devenu un enfant extrêmement savant.

\section{Tom und Julia}

1. An jenem Morgen kam die Lehrerin später als sonst in den Schulhof. Sie hatte ein kleines Mädchen an der Hand, das noch nie jemand zuvor gesehen hatte.

2. Als sie ins Klassenzimmer kam, sagte die Lehrerin: „Kinder, ich stelle euch eure neue Mitschülerin vor, sie heißt Julia. Tom, der Platz neben dir ist frei, Julia wird deine Nachbarin sein, sei nett zu ihr!"

3. Vielleicht wird Julia meine Freundin werden, dachte Tom und freute sich. Am Abend bastelte er zu Hause eine kleine rot-goldene runde Schachtel für Julia.

4. Am nächsten Morgen wartete Tom im Schulhof ungeduldig bis seine neue Nachbarin kam. Sobald er Julia sah, ging er auf sie zu und hielt ihr die Schachtel hin, die er am Vortag für sie gebastelt hatte.

5. Julia gefiel die Schachtel so gut, dass sie sie immer mit sich herumtrug. Als die Lehrerin sagte: „Packt Eure Sachen aus!“, stellte Julia die Schachtel vorsichtig zwischen Tom und sie auf ihren Tisch.

6. Eines Tages fiüsterte Julia Tom zu: „Mach die Schachtel auf!“ Tom hob den Deckel ab und entdeckte ein Stück Papier, auf das Julia geschrieben hatte: "Ich warte heute Abend um 8 Uhr unter dem großen Baum am Waldrand auf dich." 
7. Tom hatte ein bisschen Angst, weil er nicht in den Wald gehen durfte, schon gar nicht nachts.

8. Doch um 8 Uhr abends kam er dennoch zur Verabredung, Julia wartete schon auf ihn.

9. Ohne ein Wort zu sagen, nahm Julia Toms Hand und klopfte drei Mal auf den Stamm des großen Baumes.

10. Nach ein paar Minuten hörten die Kinder ein Knirschen. Der Baum fing an, sich um sich selbst zu drehen.

11. Plötzlich öffnete sich der Stamm und die Kinder wurden von dem Licht geblendet, das aus dem Inneren des Baumes kam. Sie machten einige Schritte und der Baum schloss sich hinter ihnen wieder.

12. Tom und Julia befanden sich in einem Wundergarten, wo es schien, als ob die Blumen sangen wenn sie miteinander redeten. Julia sagte nun zu Tom: „Komm, gehen wir ans andere Ende des Gartens, da wird heute Abend ein großes Fest für Dich gegeben. Bis Mitternacht darfst du dir von unserem König alles wünschen, was du willst."

13. Tom antwortete: "Ich möchte lernen, mit den Vögeln zu reden, die all das wissen, was im Himmel passiert, mit den Fischen, die all das wissen, was im Wasser passiert, und mit den Ameisen, die all das wissen, was auf der Erde passiert. "

14. Von diesem Tag an war Tom ein sehr kluges Kind. 